\title{
EFEITOS DO TREINAMENTO FÍSICO MILITAR NA APTIDÃO FÍSICA E QUALIDADE DE VIDA DE SOLDADOS
}

\section{Efects the military physical training on physical aptitude and the quality of life of the soldiers}

\author{
Marilia de Rosso Krug ${ }^{1}$ Waldomiro Eugenio Peranzoni Junior ${ }^{2}$ \\ 1,2Faculdade de Educação Física da Universidade de Cruz Alta - FEFC/UNICRUZ
}

\begin{abstract}
RESUMO: Este estudo objetivou analisar os efeitos do treinamento físico militar na aptidão física e a relação desta com qualidade de vida dos soldados incorporados no ano de 2007 na $1^{\mathrm{a}}$ bateria de obuses do $29^{\circ}$ grupo de artilharia de campanha autopropulsado de Cruz Alta/RS. Caracterizou-se como uma pesquisa do tipo descritiva. Participaram do mesmo 30 Soldados, com idade entre 19 e 20 anos, incorporados no ano de 2007 . A aptidão física foi determinada com base nos resultados obtidos nos Testes de Avaliações Físicas (TAF) que são: flexão de braço e flexão na barra (força), flexão e extensão de abdômen (RMLA) e resistência aeróbica (teste dos 12 minutos), que foram realizados 3 vezes no ano de 2007. Para determinação da percepção de qualidade de vida foi utilizado o questionário Whoqol-Bref. Os dados foram analisados através da média e desvio padrão. As diferenças entre pré e pós-testes foram estimadas através do teste "t" de Student. O Treinamento Físico Militar (TFM) melhorou, significativamente ( $\leq 0,05)$, as variáveis força (flexão de braços) e RMLA e manteve a resistência aeróbica que os soldados já possuíam antes de iniciar o referido treinamento, assim como a força (flexão na barra). A qualidade de vida dos soldados nos domínios: físico, psicológico e social apresentaram valores acima de $60 \%$ o que indica uma boa qualidade de vida. Entretanto no domínio "Meio Ambiente" os mesmos apresentaram escores baixos. A qualidade de vida geral dos soldados foi considerada "boa". Não observou-se associação significativa da aptidão física com a qualidade de vida dos soldados. Desta forma foi possível concluir que o TFM contribui para aptidão física dos soldados, sendo que a mesma não se associou significativamente com a qualidade de vida dos mesmos.
\end{abstract}

Palavras-chave: Aptidão física, Qualidade de Vida, Soldados.

ABSTRACT: This study aimed at analyzing the association between the physical aptitude and the quality of life of the soldiers incorporated on the First Battery of Obuses ( $1^{\text {a }} \mathrm{BO}$ ) in the $29^{\text {th }}$ Group of Self-propelled Artillery of Campaign ( $29^{\circ} \mathrm{GAC}$ AP) in Cruz Alta, RS, 2007 . This research is characterized as a study case. Thirty Soldiers aging 19 and 20 years old have joined this study. Their physical aptitude was determined on the basis of the results from some Physical Evaluation Tests (TAF): arm flexion and bar flexion (strength), abdominal flexion and extension (RMLA), and aerobics resistance (12 minute test), which have been applied three times during the year of 2007. The Whoqol-Bref questionnaire was used to determine the perception of quality of life of the participants. The data has been analyzed with the use of the descriptive statistics. The differences between their performances before and after-tests have been esteemed through Student's " $t$ "-test. The Military Physical Training (TFM) has improved significantly $(p \leq 0,05)$ the following variables: strength (arm flexion) and RMLA. Besides that it has kept the aerobics resistance that the soldiers already had before the beginning of the training, as well as their strength (bar flexion). The quality of life of the soldiers in terms of physical, psychological and social parameters is considered good. However in terms of "Environment" they have presented low scores. The general quality of life of the soldiers was considered "good". It was not observed in this study a significant association between the physical aptitude of the soldiers and their quality of life. Thus it is possible to conclude that the TFM has contributed for the physical aptitude of the soldiers, despite it could not be associated significantly with their quality of life.

World keys: physical aptitude, quality of life, soldiers.

Aceito em 05/07/2009 - Rev. Educ. Fís. 2009 Jun: 145: 44-49. Rio de Janeiro - RJ - Brasil

\section{INTRODUÇÃO}

Hoje todo militar considerado apto para o serviço ativo está obrigado a realizar o Treinamento Físico Militar (TFM), que visa prioritariamente à preservação da saúde (qualidade de vida) e permitir ao mesmo que atinja padrões para o desempenho físico compatíveis com a operacionalidade desejada.
A preparação da condição física total dos militares é desenvolvida através de atividades físicas variadas, regulares e controlada, realizada diariamente, que seguem um quadro de atividades semanais tais como: exercícios de resistência aeróbica e de resistência muscular localizada, que deverão contribuir para a melhora da aptidão física dos soldados ${ }^{(1)}$.

A aptidão física é composta por aspectos biológicos 
(antropométricos, metabólicos e neuro-musculares) sendo que estes fatores apresentam uma organização dinâmica no ser humano e conceitualmente podem voltar-se tanto para o bem estar geral e uma boa saúde, como para uma melhor qualidade de vida(2).

A influência do exercício físico sobre a qualidade de vida se dá sob diversos aspectos, um deles é a ação benéfica que ele exerce sobre os efeitos do estresse cotidiano o que proporciona um melhor gerenciamento das tensões próprias do dia-a-dia, pois na prática do exercício físico ocorre uma descarga fisiológica de energia acumulada através do corpo, podendo desta forma fazer com que o militar evite que se crie um estado de agitação e desassossego interior tremendamente nocivo ao bem estar, a felicidade e a alegria do individuo ${ }^{(3)}$.

Atualmente o enfoque do treinamento físico militar deve atender de melhor forma a saúde e aos interesses do militar, sendo assim seu bem estar, tendo objetivos e benefícios mais duradouros no tempo e proporcionando uma melhor qualidade de vida(1). Desta forma justifica-se este estudo que teve como objetivo: "analisar os efeitos do treinamento físico militar na aptidão física e a relação desta com qualidade de vida dos soldados incorporados no ano de 2007 na $1^{\text {a }}$ bateria de obuses do $29^{\circ}$ grupo de artilharia de campanha autopropulsado de Cruz Alta/RS.

\section{METODOLOGIA}

Participaram desse estudo os soldados incorporados no ano de 2007 na $1^{\text {a }}$ Bateria de Obuses do $29^{\circ}$ Grupo de Artilharia de Campanha Autopropulsado, sendo um total de 30, com idade entre 19 e 20 anos. No estudo foi preservado suas identidades, como forma de respeito as suas individualidades. Todos assinaram um termo de consentimento livre e esclarecido.

A aptidão física dos soldados foi determinada com base nos resultados obtidos nos Testes de Avaliações Físicas (TFM), propostas pelo exército, que foram realizados três vezes no ano de 2007, respectivamente nos meses de Abril, Agosto e Novembro, englobando os seguintes testes: flexão de braço e flexão na barra (força), flexão e extensão de abdômen (RML) e resistência aeróbica (teste dos 12 minutos). Os testes foram realizados na parte da manhã.

A flexão de braço foi realizada em quatro apoios, na execução do teste o cotovelo ultrapassou a linha do tronco.

A flexão na barra foi realizada na vertical, onde o executante ultrapassou o seu queixo da linha da barra.

O teste de flexão e extensão de abdômen foi realizado através do exercício "abdominal supra".

A resistência aeróbica foi obtida através do teste de correr/andar 12 minutos, onde o executante percorreu o máximo de distância possível no tempo destinado, este teste é um dos mais utilizados no meio militar, principalmente pela sua facilidade de aplicação.

Para identificação da qualidade de vida, que foi definida pelo Grupo de Qualidade de Vida da Organização Mundial da Saúde como "a percepção do indivíduo de sua posição na vida, no contexto da cultura e sistema de valores nos quais ele vive e em relação aos seus objetivos, expectativas, padrões e preocupações"(4). Foi utilizado o questionário WHOQOL-BREF.

Apesar da crescente importância da avaliação da qualidade de vida em diferentes áreas da medicina, não havia nenhum instrumento desenvolvido dentro de uma perspectiva transcultural para uso internacional. Assim, inicialmente foi desenvolvido um instrumento de avaliação de qualidade de vida com 100 questões (o WHOQOL-100). O desenvolvimento do WHOQOL-100 seguiu metodologia descrita em outras publicações envolvendo a participação de vários países, representando diferentes culturas ${ }^{(5)}$. Entretanto a necessidade de instrumentos curtos que demandem pouco tempo para seu preenchimento, mas com características psicométricas satisfatórias, fez com que o Grupo de Qualidade de Vida da OMS desenvolvesse uma versão abreviada do WHOQOL-100, o WHOQOLbref $^{(4)}$. O mesmo consta de 26 questões, sendo duas questões gerais de e as demais 24 representam cada 
uma das 24 facetas que compõe o instrumento original. Assim, diferente do WHOQOL-100 em que cada uma das 24 facetas é avaliada a partir de quatro questões, no WHOQOL-bref é avaliada por apenas uma questão. Os dados que deram origem à versão abreviada foram extraídos do teste de campo de 20 centros em 18 países diferentes.

O Treinamento Físico Militar (TFM) foi realizado semanalmente por todos militares, ao longo de todo ano de 2007, possuindo no mínimo dois tempos de instrução. O Oficial de Treinamento Físico Militar (OTFM) juntamente com o Chefe da $3^{a}$ Seção (Seção de Operações) da Organização Militar (OM), responsável pela instrução da OM prescreveu para cada dia da semana um tipo de atividade física, na qual englobava atividades de resistência aeróbica (corrida contínua), força (séries de flexões de braços e flexões na barra) e resistência muscular localizada de abdômen (séries de abdominais).

Os dados foram analisados com a utilização do programa Microsoft Excel e SPSS 10.0, sendo descrito em função de sua média e desvio padrão. As diferenças entre pré e pós-testes foram estimadas através do Teste " $t$ " de Student.

A correlação linear de Pearson foi utilizada para determinação da existência de correlação entre a aptidão física e a qualidade de vida dos soldados. 0 nível de significância adotado foi de $p \leq 0,05$.

\section{RESULTADOS E DISCUSSÕES}

Na TABELA 1 encontram-se os resultados médios e desvio padrão das variáveis dos Testes de Avaliação Física (TAF).

Analisando os dados, da TABELA 1, notou-se diferenças estatisticamente significativas $(p \leq 0,05)$ somente para as variáveis força (flexão de braços) e RMLA, ou seja, o Treinamento Físico Militar (TFM) aumentou a força e RMLA e somente manteve a resistência aeróbica que os soldados já apresentavam antes de iniciar o referido treinamento, assim como a força (flexão na barra).
TABELA 1

DADOS MÉDIOS E DESVIO PADRÃO DAS VARIÁVEIS DO TAF.

\begin{tabular}{cccc}
\hline VARIÁVEIS & TAF I & TAF II & TAF III \\
\hline Resistência Aeróbica $\left(\right.$ VO $_{2}$ máx) & $55,00 \pm 5,80^{\mathrm{a}}$ & $55,17 \pm 4,23^{\mathrm{a}}$ & $55,93 \pm 4,44^{\mathrm{a}}$ \\
Força (Flexão de braço) & $34,73 \pm 6,89^{\mathrm{a}}$ & $39,78 \pm 5,28^{\mathrm{b}}$ & $38,47 \pm 6,04^{\mathrm{b}}$ \\
Força (flexão na barra) & $7,21 \pm 3,50^{\mathrm{a}}$ & $7,86 \pm 2,21^{\mathrm{a}}$ & $8,23 \pm 2,48^{\mathrm{a}}$ \\
RMLA & $69,42 \pm 17,46^{\mathrm{a}}$ & $87,13 \pm 13,71^{\mathrm{a}}$ & $79,47 \pm 10,33^{\mathrm{b}}$ \\
\hline
\end{tabular}

a,bMédias seguidas de letras diferentes diferem entre si $(p \leq 0,05)$.

Tanto a resistência aeróbica quanto a força (flexão na barra) mantiveram-se em um conceito "Bom", de acordo com a Portaria $N^{\circ} 223-\mathrm{EME}^{(6)}$ que aprova a diretriz para o treinamento físico militar do exército e a sua avaliação.

A força (flexão de braço) teve um ganho, significativo, do "TAF I" para o "TAF II", evoluindo do conceito "Bom" para o "Excelente" (6) e se manteve do "TAF II" para o "TAF III".

A RMLA diminuiu, significativamente, do "TAF II" para o "TAF III", entretanto manteve as duas médias no conceito "Excelente", sendo que a média do "TAF I" era "Muito Bom" (6).

Com base nestes resultados pode-se dizer que a aptidão física dos soldados encontrava-se no conceito "Bom" (6).

Dos testes adotados pelo Exército, a flexão de braços e o abdominal (ainda que com detalhes de execução diferente da forma solicitada no TAF) são freqüentemente utilizados para medir resistência muscular localizada de membros superiores e abdômen, respectivamente(7) e a corrida de 12 minutos como apropriada para resistência aeróbia.

A resistência aeróbica é uma definição comum do componente funcional que se refere à função cardiovascular e pulmonar, sendo entendida como uma capacidade do corpo de manter um exercício submáximo durante períodos prolongados de tempo. O desempenho aeróbico é considerado prioritário à promoção da saúde e também ao desempenho atlético. As atividades que intervêm em grandes grupos musculares e atuam sobre o sistema 
cardiovascular e respiratório são prioritárias dessa qualidade física(8).

Uma excelente resistência cardiorrespiratória reflete em um coração forte, bons vasos sangüíneos e correto funcionamento dos pulmões. Quanto melhor for essa capacidade, melhor será a aptidão física do individuo e mais rápida será a recuperação após a

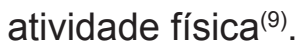

A força e a resistência muscular localizada referemse à capacidade de um músculo, ou de um grupo muscular, de sustentar varias contrações repetidas por um determinado período de tempo. Índices adequados de força/resistência muscular localizada previnem problemas posturais, articulares e lesões músculoesquelético. "Debilidades, nesses componentes indicam riscos de lombalgia e fadiga localizada" (8).

Uma musculatura corporal fortalecida pode reduzir em grande chance a probabilidade de ocorrer entorses, rupturas musculares e outras lesões tradicionais de quem pratica regularmente atividade física ${ }^{(9)}$.

A força e a resistência muscular localizada prejudicadas podem acarretar um desencadeamento de graves distúrbios músculos-esqueléticos, que resultam em dores e desconfortos consideráveis ${ }^{(10)}$.

Em um ambiente militar, a força/resistência muscular localizada é consideravelmente importante, cargas típicas carregadas por soldados incluem munição, armamento, equipamento individual e demais materiais necessários para o cumprimento da missão, o peso desses materiais é sempre o mesmo, independentemente da força individual do soldado, logo, soldados mais fortes e resistentes terão uma maior capacidade para suportar tais $\operatorname{cargas}^{(8)}$.

Para determinar a qualidade de vida dos soldados foi utilizado o questionário de percepção da qualidade de vida - Whoqol-Bref, proposto pela Organização Mundial da Saúde (OMS) possuindo 26 questões, sendo composto por quatro domínios: Físico, Psicológico, Relações Sociais e Meio ambiente. O mesmo é analisado em uma escala de zero (0), péssima qualidade de vida, a cem (100) excelente qualidade de vida.

TABELA 2

QUALIDADE DE VIDA DOS SOLDADOS.

\begin{tabular}{cc}
\hline Domínios & $\begin{array}{c}\text { Média } \pm \text { desvio } \\
\text { padrão }\end{array}$ \\
\hline Físico & $78,21 \pm 11,75$ \\
Psicológico & $75,83 \pm 10,79$ \\
Social & $76,38 \pm 16,09$ \\
Ambiente & $59,68 \pm 10,19$ \\
Qualidade de vida geral & $71,76 \pm 09,07$ \\
\hline
\end{tabular}

Analisando os dados da TABELA 2, pode-se perceber que a percepção de qualidade de vida dos soldados nos domínios físico, psicológico e social é melhor em relação ao domínio meio ambiente.

Desta forma pode-se inferir que no meio ambiente em que os soldados trabalham existe algumas imposições que podem estar relacionadas como hierarquia sobre eles e a disciplina na qual eles devem ter com o seu superior, com o seu subordinado ou com seus pares, estes aspectos às vezes influencia e muito no desempenho

TABELA 3

CORRELAÇÃO ENTRE APTIDÃO FÍSICA E QUALIDADE DE VIDA (R).

\begin{tabular}{ccccc}
\hline QV & $\begin{array}{c}\text { Força } \\
\text { (Flexão de braços) }\end{array}$ & $\begin{array}{c}\text { Força } \\
\text { (Flexão na barra) }\end{array}$ & RML & VO $_{2}$ máx \\
\hline D. Físico & 0,08 & 0,04 & $-0,27$ & 0,03 \\
D. Psicológico & 0,12 & 0,22 & 0,08 & 0,14 \\
D. Social & 0,31 & 0,67 & 0,44 & 0,09 \\
D. Ambiental & $-0,04$ & $-0,00$ & 0,27 & 0,13 \\
QV Geral & $-0,10$ & 0,08 & $-0,16$ & 0,18 \\
\hline
\end{tabular}


profissional. Mesmo a qualidade de vida no domínio meio ambiente não tendo apresentado um escore muito alto a qualidade de vida geral dos soldados foi considerada "boa".

As variáveis da aptidão física (força, RML e consumo máximo de oxigênio $\mathrm{VO}_{2}$ máx) foram correlacionadas (correlação linear de Pearson) com os domínios da qualidade de vida (DQV) e com a qualidade de vida geral (TABELA 3).

Observando os resultados da TABELA 3 foi possível notar que não existe correlação significativa entre a aptidão física e a qualidade de vida dos soldados.

Embora neste estudo não tenha ocorrido correlação significativa, estudos epidemiológicos e documentos institucionais propõem que a prática regular de atividade física e uma boa aptidão física estão associadas a uma menor mortalidade e melhor qualidade de vida em população jovem. Existem cada vez mais dados demonstrando que o exercício, a aptidão e a atividade física estão relacionados com a prevenção, com a reabilitação de doenças e com a qualidade de vida(11).

Uma boa condição muscular proporciona uma maior capacidade de realizar atividades físicas melhorando assim a auto-estima e o sistema emocional do indivíduo ${ }^{(12)}$.

"A atividade física regular é de extrema importância na saúde psicológica, social e fisiológica das pessoas"(13).

\section{CONCLUSÃO}

Após a análise dos dados foi possível concluir que o Treinamento Físico Militar (TFM) influenciou na aptidão física dos soldados somente nas variáveis força (flexão de braços) e RMLA, e manteve a resistência aeróbica que os soldados já possuíam antes de iniciar o referido treinamento, assim como a força (flexão na barra) e estas variáveis não apresentaram associação,significativa com a qualidade de vida dos soldados.
A qualidade de vida dos soldados nos domínios: físico, psicológico e social foi considerada boa. Entretanto no domínio "Meio Ambiente" os mesmos apresentaram escores baixos. Mesmo a qualidade de vida no domínio meio ambiente não tendo apresentado um escore muito alto a qualidade de vida geral foi boa.

\section{REFERÊNCIAS}

1. Brasil. Ministério da Defesa. Exército Brasileiro. Estado Maior do Exército. Manual de Campanha: Treinamento Físico Militar; C 20-20. 3. ed. Brasília - DF, 2002.

2. Matsudo VKR. Testes em ciências do esporte. São Caetano do Sul: CELAFISCS, 1998.

3. Silva MAD da. Exercício e Qualidade de Vida. In: Ghorayeb NB, Turíbio B. O Exercício: Preparação Fisiológica, Avaliação Médica, Aspectos Especiais e Preventivos. São Paulo: Atheneu, 1999.

4. The Whoqol Group. The development of the World Health Organization quality of life assessment instrument (the WHOQOL). In: ORLEY J, KUYKEN W, editors. Quality of life assessment: international perspectives. Heidelberg: Springer Verlag;1994; 1:41-60.

5. Fleck MPA, Fachel O, Louzada S, Xavier M, Chachamovich E, Vieira G, et al. Projeto desenvolvido para a OMS no Brasil pelo grupo de estudos em qualidade de vida. Divisão de Saúde Mental Grupo Whoqol Versão em Português dos Instrumentos de Avaliação de Qualidade de Vida (Whoqol) 1998 OMS do BR- Set.1998. Universidade Federal do Rio Grande Do Sul. Porto Alegre, RS 1998. Disponível em: <http://www.ufrgs.br/Psiq/whoqol1.html>. Acesso em: 16 ago. 2008.

6. Brasil. Ministério da Defesa. Exército Brasileiro. Secretaria-Geral do Exército. Boletim do Exército $N^{\circ}$ 01/2006, Brasília - DF, 6 de janeiro de 2006, Portaria N ${ }^{\circ}$ 223-EME, de 23 de Dezembro de 2005. Brasília - DF, 2005.

7. Carnaval PE. Medidas e avaliação em ciências do esporte. Rio de Janeiro: Sprint, 1995.

8. Pereira ÉF, Teixeira CF. Proposta de valores normativos para avaliação da aptidão física em militares da Aeronáutica. Revista Brasileira Educação Física e Esportes, São Paulo 2006,4:249-56. Disponível em: <http://www.usp.br/eef/rbefe/ vol20_n4/3_v20_n4_p249.pdf>. Acesso em 08 maio 2008. 
9. Glaner MF. Importância da aptidão física relacionada à saúde. Rev Bras Cineantropom Desempenho Hum 2003;2:75-85.

10. Pollock ML, Wilmore JH. Exercícios na saúde e na doença: avaliação e prescrição para reabilitação. 2. ed. Rio de Janeiro: MEDSI, 1993.

11. Araújo DSMS, Araújo GS. de; Aptidão física, saúde e qualidade de vida relacionada à saúde em adultos. Reva Bras MedEsporte 2000;5:193-203.

12. Nahas MV. Atividade Física, saúde e qualidade de vida: conceitos e sugestões para um estilo de vida ativo. 3. ed. Londrina: Midiograf, 2003.

13. Pontes Junior, FL. Envelhecimento. In: Corazza, MA. Terceira idade e atividade física. São Paulo: Phorte, 2001.
Endereço para correspondência:

Marilia de Rosso Krug

Rua: Coronel Niederauer, 1537, Apto 702

Bairro: Centro

CEP: $97015-123$

Santa Maria/RS

Fones (55) 33117558 ou 91492701

e-mail: mariliakrug@bol.com.br

Waldomiro Eugenio Peranzoni Junior Rua: Miguel Carvalho de Macedo, 45 Bairro Goianias

CEP 97070-530

Fones (55)32111344 ou (55)99719561

e-mail: tenperanzoni@hotmail.com 